\title{
Deadbands, Droop and Inertia Impact on Power System Frequency Distribution
}

\author{
Petr Vorobev, Member, IEEE, David M. Greenwood, Member, IEEE, John H. Bell, \\ Janusz W. Bialek, Fellow, IEEE, Philip C. Taylor, Senior Member, IEEE, and Konstantin Turitsyn, Member, IEEE
}

\begin{abstract}
Power system inertia is falling as more energy is supplied by renewable generators, and there are concerns about the frequency controls required to guarantee satisfactory system performance. The majority of research into the negative effect of low inertia has focused on poor dynamic response following major disturbances, when the transient frequency dip can become unacceptable. However, another important practical concern keeping average frequency deviations within acceptable limits - was mainly out of the sight of the research community. In this manuscript we present a method for finding the frequency probability density function (PDF) for a given power system. We pass from an initial stochastic dynamic model to deterministic equations for the frequency PDF, which are analyzed to uncover key system parameters influencing frequency deviations. We show that system inertia has little effect on the frequency PDF, making virtual inertia services insufficient for keeping frequency close to nominal under ambient load fluctuations. We establish, that aggregate system droop and deadband width are the only parameters that have major influence on the average frequency deviations, suggesting that energy storage might be an excellent solution for tight frequency regulation. We also show that changing the governor deadband width does not significantly affect generator movement..
\end{abstract}

Index Terms-Frequency control, droop control, deadbands, low inertia grids, frequency fluctuations.

\section{INTRODUCTION}

In electric power systems, primary and secondary frequency controls are aimed at maintaining a stable system frequency and restoring power balance [1]. During any major disturbance (typically a loss of a generating unit) frequency deviates rapidly from the nominal, triggering all generators participating in primary response to quickly readjust their output and restore the power balance. However, even during prefault operation, frequency can drift from its nominal value due to stochastic load fluctuations, and generators participating in frequency control constantly readjust their power output in response. The ability of the system to keep frequency within

This work was supported in part by Skoltech-MIT Next Generation program, in part by MIT Undergraduate Research Opportunities Program (UROP), and in part by the Engineering and Physical Sciences Research Council (EPSRC) under grant number EP/K002252/1

P.Vorobev and J. W. Bialek are with the Skolkovo Institute of Science and Technology (Skoltech), Moscow, Russia E-mail: p.vorobev@skoltech.ru, J.Bialek@skoltech.ru

J.H.Bell is with Department of Mechanical Engineering, Massachusetts Institute of Technology. E-mail: jhbell@mit.edu

D.M. Greenwood and P.C.Taylor are with the School of Electrical and Electronic Engineering, Newcastle University, Newcastle Upon Tyne, U.K. Email: : david.greenwood@ncl.ac.uk; phil.taylor@ncl.ac.uk

K.Turitsyn was with Department of Mechanical Engineering,Massachusetts Institute of Technology. E-mail: turitsyn@gmail.com certain limits the majority of the time is one of the major performance characteristics of a power system.

A number of metrics exist to assess frequency regulation adequacy; one of the most cited sets of metrics is related to transient frequency response following a contingency [2]. The main performance indices considered are the rate of change of frequency (ROCOF), the frequency nadir - the maximum frequency drop following a contingency - and a quasi-steady state frequency deviation [3], [4]. The three major system parameters which affect these indices are: overall system inertia, effective droop coefficient, and governor/turbine delays. The system inertia is by far the most discussed parameter in research literature due to the fact that, unlike the droop response, it can not be manually tuned; instead, it is determined by the amount of rotating mass that is synchronously coupled to the system.

Overall system inertia is considered to be one of the major factors affecting both ROCOF and frequency nadir during a transient [3], [5], [6], and decreasing system inertia is one of the main parameters limiting further increases in renewable penetration [7], [8]. Numerous papers are dedicated to renewable energy resources participating in frequency control, mostly focusing on wind turbines interfaced with doubly fed induction machines [9]-[11]. Such a synthetic inertial and droop response, however, requires continuous operation of the renewable generator below the maximum power point, which constitutes a non-zero cost of such services.

Transient frequency response following major disturbances is not the only metrics that should be considered when assessing frequency regulation adequacy. The system should also be able to keep the average frequency deviation over extended periods of time within certain limits. Thus, the so-called Control Performance Standards (CPS1 and CPS2) [12] issued by NERC in late 90 s imposed a limit on a root mean square (RMS) frequency deviation over a one year period; a detailed description, with particular target values, can be found in [13]. While the influence of frequency control settings and power systems parameters on the transient frequency response has been extensively studied, there is still little research available on how those settings affect the RMS frequency deviation. One of the reasons is that dynamic equations with stochastic terms should be considered in order to analyze the average frequency deviations. Moreover, unlike for transient frequency response, where a single dynamic simulation can provide enough data, multiple simulations or a single simulation over an extended period of time are required to get statistically valid results.

A comprehensive framework for modelling power sys- 
tem dynamics with stochastic perturbations represented by an Ornstein-Uhlenbeck process was developed in [14]. The method was then applied to perform a number of simulations for transient stability assessment. In a later publication [15] the authors offered a method for obtaining the noise parameters of the load using micro-PMU (Phasor Measurement Unit) measurement data. In [16] a direct simulation based on stochastic model was performed to study the frequency probability distribution function and the results were compared to real-life data for Irish power system. In a recent study [17] power system response to random ambient fluctuations, which can be obtained from PMU data, was used to estimate the dynamic frequency response at different buses following a localized disturbance. Finally, the series of works [18]-[20] presented a study of the compliance of Texas power system (ERCOT) automatic generation control settings to NERC CPS standards.

Instead of running lengthy numerical simulations, one can derive an explicit equation for the probability density function (PDF) of system frequency. Under the assumption, that the underlying load fluctuations can be modelled as white noise, the derivation is straightforward and leads to the, so-called, Fokker-Planck equation. Such an approach was used in [21] for probabilistic assessment of power system transient stability. While the use of Fokker-Planck equation requires certain assumptions about the noise type, it allows one to explicitly find the probability distribution functions for different system states bypassing the need for lengthy direct numerical simulations. Thus, in [22] the performance of different primary frequency control methods in the presence of random load fluctuations and measurement noise was analyzed by calculating RMS frequency deviation. Moreover, for linear system representation, a semi-analytic solution is available which allows for explicit analysis of the influence of different system parameters on the probability distribution functions of it's states. In an early paper [23] statistical analysis was performed to compare CPS1 and CPS2 standards assuming Gaussian form for probability distribution function of frequency.

In this manuscript we apply the Fokker-Planck equation to study probability density functions of frequency deviations in power systems. We explicitly study the influence of inertia, droop coefficients, secondary frequency control settings, turbine time-constant and governor deadbands on the distribution function of frequency deviations. Based on our analysis we make predictions on how the frequency deviations will change in future systems with more renewable generation, and suggest possible ways of improving the performance of frequency control.

The main contributions of the present manuscript are as follows:

1) A Fokker-Planck equation for the frequency PDF is derived and explicitly solved. The influence of system parameters on the frequency PDF is explicitly established and analyzed.

2) Inertia is shown to have little effect on the RMS frequency deviation, contrary to it's role in transient frequency response to contingencies.
3) We demonstrate the effect of governor deadbands, showing that simultaneous decrease in deadbands across the whole system reduced the RMS frequency deviation without leading to significantly higher generator wearand-tear.

\section{Modelling ApPROACH}

In this section and Section III we describe our general approach to studying the long-term frequency distribution in power systems. Our main purpose is to establish a model that enables the study of how different system parameters and control settings affect the long-term frequency distribution. We start from the general dynamic model with stochastic perturbations, which is then used to derive an equation for the frequency deviations probability density function (PDF). We initially consider a power system with the vector of its states denoted as $\mathbf{x}(t)$ (with $N$ dimensions), for which we write the set of dynamic equations in a general form:

$$
\dot{\mathbf{x}}(t)=\boldsymbol{\mu}(\mathbf{x}, t)+\mathbf{B} \boldsymbol{\xi}(t)
$$

here $\boldsymbol{\mu}(\mathbf{x}, t)$ represents the deterministic part of the power system dynamics, and is usually referred to as the drift term (in general it is a non-linear function of state vector $\mathbf{x}$ with explicit dependence on time). Stochastic perturbations are characterized by the diffusion term $\mathbf{B} \boldsymbol{\xi}(t)$ where $\mathbf{B}$ is an $N \times M$ matrix and $\boldsymbol{\xi}(\boldsymbol{t})$ is the $M$-dimensional vector of white noise (which can be defined as the time derivative of the $M$ dimensional Wiener process) with the following properties:

$$
\mathbb{E}\left[\xi_{i}(t)\right]=0 ; \quad \mathbb{E}\left[\xi_{i}\left(t_{1}\right) \xi_{k}\left(t_{2}\right)\right]=\delta_{i k} \delta\left(t_{1}-t_{2}\right)
$$

where $\delta_{i k}$ is the Kronecker delta and $\delta(t)$ is the Dirac deltafunction.

One can directly simulate the dynamics caused by the system (1) (this system in an even more general form was also reported in [14] and [16]) with some generated white noise signal and then infer the distribution function for the system states from the results of these simulations. However, large, computationally intensive simulations would be needed to obtain a sufficiently smooth distribution functions. Moreover, such a direct numerical method will provide limited insight into the system. As an illustration, Fig 1 provides a histogram for frequency deviations in the Great Britain (GB) power system for two separate days - the source is $1 s$ resolution frequency measurements data available from National Grid [24]. It is evident that on a one-day horizon frequency deviations do not provide statistically reliable picture - the two histograms in Fig 1 are very different. On the other hand, averaging over onemonth period is sufficient, which is illustrated by the Fig. 2 where the histograms of frequency deviations for two different months look almost identical.

A natural way of dealing with the shortcomings of direct numerical simulations is to consider probability density function (PDF) $\mathcal{F}(\mathbf{x}, t)$ for system states. Under the assumption that the noise in (1) is white, it is possible to write a deterministic equation for the PDF. Following standard rules one can derive the Fokker-Planck equation for $\mathcal{F}(\mathbf{x}, t)$ [25]:

$$
\frac{\partial}{\partial t} \mathcal{F}(\mathbf{x}, t)=-\nabla^{T}[\boldsymbol{\mu} \mathcal{F}(\mathbf{x}, t)]+\nabla^{T} \mathbf{D} \nabla \mathcal{F}(\mathbf{x}, t)
$$




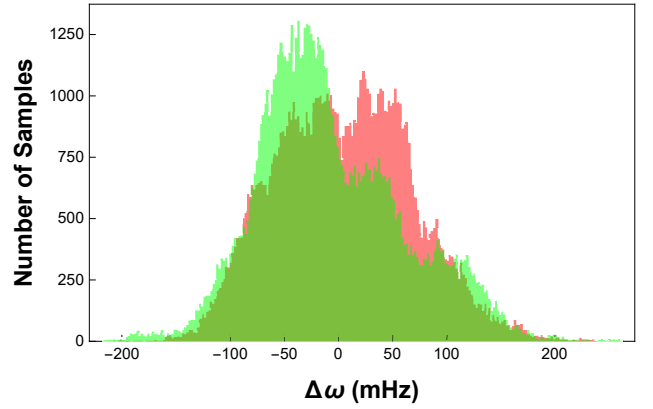

Fig. 1. Histograms of measured frequency deviations in GB power grid for two different days: day 1 - green, day 2 - red.

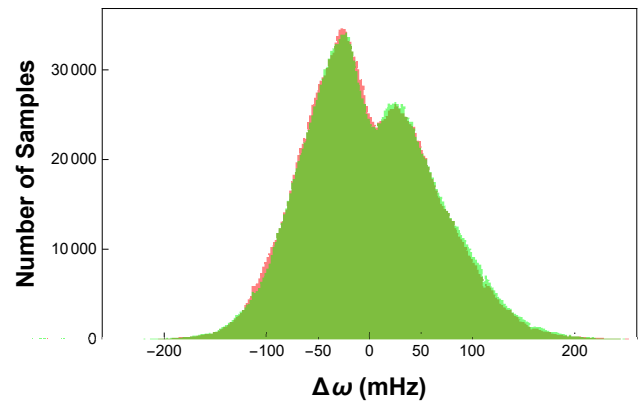

Fig. 2. Histograms of measured frequency deviations in GB power grid for two consecutive months, marked by green and red. As can be seen, two histograms almost coincide.

where $\nabla$ is the Nabla-operator over the space of system states and $\mathbf{D}$ is the diffusion matrix defined as $\mathbf{D}=\mathbf{B B}^{T} / 2$.

Solution of equation (3) provides a PDF of all system states $\mathrm{x}$ as a function of time. For finding probability density of a certain state over a long period of time, a stationery form of equation (3) with zero left-hand side can be used:

$$
\nabla^{T} \mathbf{D} \nabla \mathcal{F}(\mathbf{x}, t)-\nabla^{T}[\boldsymbol{\mu} \mathcal{F}(\mathbf{x}, t)]=0
$$

In the present manuscript we are interested in the statistical behaviour of frequency deviations on the timescales of months (eg. Fig 2), therefore equation (4) will be used throughout the manuscript.

Both equations (3) and (4) are in general non-linear and solving them for a large system can be very difficult. However, both these equation can be efficiently solved for a system of arbitrary size in a very important case when the drift term $\boldsymbol{\mu}(\mathbf{x}, t)$ can be represented as linear function of system states $\mathbf{x}$ with some constant state matrix $\mathbf{A}$. In this case the corresponding dynamic equations (11) reduce to:

$$
\dot{\mathbf{x}}(t)=\mathbf{A} \mathbf{x}+\mathbf{B} \boldsymbol{\xi}(t)
$$

If the dynamic system (5) is stable (which we assume, as we are investigating the pre-fault frequency distributions) then the stationery equation (44) has solution of the form [25]:

$$
\mathcal{F}(\mathbf{x})=(\operatorname{det}|2 \pi \mathbf{C}|)^{-1 / 2} \cdot \exp \left(-\frac{1}{2} \mathbf{x}^{T} \mathbf{C}^{-1} \mathbf{x}\right)
$$

where the co-variance matrix $\mathbf{C}$ is symmetric positive definite and satisfies the following equation:

$$
\mathbf{A C}+\mathbf{C A}^{T}=-\mathbf{D}
$$

This matrix equation can be solved numerically for a system of arbitrary dimensions. For the systems with smaller number of states an analytic solution is possible.

It is true that, in general, the dynamics of a power system is non-linear. However, linear approximation can be effectively used if the magnitudes of perturbations are small. As is seen from the Fig 2, most of the time frequency fluctuations are within $\pm 100 \mathrm{mHz}$, which is around $0.2 \%$ of the nominal value. Since in this study we are interested in frequency statistical properties, rather than separate large-deviation events, it is justified to start form a linearized dynamic model, since it provides an insight into the problem. Therefore, in Section III] we will assume linear frequency dynamics, validation of our findings on fully non-linear models will be performed in Section IV

\section{SOlution OF FoKker-Planck EQuation}

In this section we provide a detailed analysis of the solution of the Fokker-Planck equation for different system models - we explicitly consider the effects of inertia, turbine time-constant, secondary control and deadbands. We establish the main parameters affecting the frequency deviations and provide references to possible practical outcomes of our findings.

To study frequency fluctuations, we will consider only the dynamics of the center of inertia (COI) of a power system, which is justified by the fact that frequency dynamics timescales (starting at several seconds) are much larger than the timescales of inter-area modes as well as dynamic phenomena associated with high-order generator models [26]. The adequacy of this single area (or single bus) model for frequency dynamics representation was confirmed by numerous studies (for eg. [6], [27], [28]). In Section IV] we will perform the validation on a bigger system.

\section{A. Basic Model}

We start from a simple model, in which a power system with only primary frequency control is considered: governor deadbands, turbine time-constants and secondary frequency control are assumed to be absent in this initial model. We assumed that the load stochastic dynamics can be represented as an Ornstein-Uhlenbeck Process (OUP) [14], [16], [29], [30]. Under these assumptions the system dynamic equations are:

$$
\begin{aligned}
& 2 H \dot{u}=-\alpha u-p \\
& \dot{p}=-\frac{1}{\tau_{p}} p+b \xi
\end{aligned}
$$

where we used the following denotations: $H$ - is the system aggregate inertia time constant, $u$ - per unit frequency deviation from the nominal (i.e. $\left.u(t)=\left(\omega(t)-\omega_{0}\right) / \omega_{0}\right), \alpha=1 / R$ is the inverse aggregate dimensionless droop coefficient of the system (including both generator response and load sensitivity to frequency), $p$ - per unit load deviation from the base value, $\tau_{p}$ is the load fluctuations mean reversal time and $b$ is the noise amplitude. Instead of $b$ it is more convenient to use the, so-called, diffusion coefficient $D=b^{2} / 2$. [29].

The stationary Fokker-Planck equation for the combined frequency and power probability density function $\mathcal{F}(u, p)$ 
corresponding to the stochastic system $(8)$ can be derived using (4):

$$
D \frac{\partial^{2} \mathcal{F}}{\partial p^{2}}+\frac{1}{\tau_{p}} \frac{\partial(p \cdot \mathcal{F})}{\partial p}+\frac{1}{2 H} \frac{\partial}{\partial u}[(\alpha u+p) \mathcal{F}]=0
$$

This equation can be explicitly solved to provide a normal distribution for $\mathcal{F}(u, p)$ :

$$
\mathcal{F}(u, p)=\frac{1}{2 \pi \kappa \chi} \exp \left[-\frac{(p+\alpha u)^{2}}{2 \kappa^{2}}-\frac{u^{2}}{2 \chi^{2}}\right]
$$

with variances $\kappa^{2}$ and $\chi^{2}$ given by:

$$
\kappa^{2}=\frac{2 \tau_{p} D H}{2 H+\alpha \tau_{p}} ; \quad \chi^{2}=\frac{\tau_{p}^{2} D}{\alpha\left(2 H+\alpha \tau_{p}\right)}
$$

Function $\mathcal{F}(u, p)$ in (10) gives the simultaneous probability density for frequency and load deviations $u$ and $p$. The probability density function for frequency only - $F(u)$ - can be found from $\mathcal{F}(u, p)$ by means of integration over $p$ :

$$
F(u)=\int_{-\infty}^{+\infty} \mathcal{F}(u, p) d p
$$

Performing integration of (10) over all values of $p$ we find:

$$
F(u)=\frac{1}{\sqrt{2 \pi \sigma^{2}}} e^{-\frac{u^{2}}{2 \sigma^{2}}}
$$

with the following closed-form expression for frequency standard deviation:

$$
\sigma=\left(\frac{D \tau_{p}^{2}}{\alpha\left(2 H+\alpha \tau_{p}\right)}\right)^{1 / 2}
$$

The value of this solution is that it allows one to calculate the RMS frequency deviation, provided parameters of the power system are known. In practice, the aggregate inertia $H$ and inverse droop $\alpha$ are readily available; conversely, both load mean reversal time $\tau_{p}$ and diffusion coefficient $D$ are difficult to measure directly. However, one can use the formula (14) to predict the effect of the change in inertia and/or aggregate droop on the frequency deviations provided the loading conditions do not change significantly.

The denominator of expression (14) can be further simplified by considering realistic estimations for the system parameters: inertia term $H$ is around $5-6 s$ or smaller for reallife systems [6], [31], while $\alpha$ - the inverse droop - is typically in the interval of $\sim 10-20$ depending on the number of units directly participating in primary response [32]. Therefore, the inertial term in the denominator of (14) is small if the load mean reversal time $\tau_{p}$ is significantly greater than $\sim 1 s$. Different estimations for $\tau_{p}$ are cited in literature [14]-[16], most of them being well above $1 \mathrm{~s}$. An approximate estimation for the lower bound on $\tau_{p}$ can be inferred from considering an average duration of either low or high frequency events from historical data. Thus, an average duration of about $\sim 30 \mathrm{~s}$ was cited for US Eastern Interconnection in [33], and a similar value was obtained in [34 from analysis of the GB system data. Therefore, in the following we will assume that condition $\alpha \tau_{p} \gg H$ is satisfied. Under this assumption the expression (14) can be further simplified:

$$
\sigma=\frac{\sqrt{D \tau_{p}}}{\alpha}
$$

In this form, the RMS frequency deviation appears to be independent of the system inertia. Another way of stating this fact is to calculate the sensitivity of the frequency standard deviation to changes in the inertia value, given by the formula:

$$
\nu_{H}=\frac{d \sigma}{d H}
$$

Differentiating equation (14) we find that $\nu_{H}=-0.19 \mathrm{mHz} / \mathrm{s}$ (for the values of power system parameters from Table \.This means that the change of the system inertia constant by $1 \mathrm{~s}$ leads to the change of RMS frequency deviation only by $0.19 \mathrm{mHz}$ in the opposite direction. To avoid confusion, we once again emphasize that we are only considering the frequency fluctuations under the ambient load perturbations. In the case of a sudden major disturbance (such as a loss of a generating unit), inertia is one of the most important parameters determining the depth of the frequency dip, and has already been shown to be one of the main limiting factors for renewables penetration [2], [4].

Determining the real-life values of system-wide diffusion $D$ and load reversal time $\tau_{p}$ would require observation of the total load fluctuations over the extended period of time, and subsequent statistical analysis. According to (15) the values of both parameters can not be obtained from the data on the system frequency only. However, the product $D \cdot \tau_{p}$ can be inferred from the measurements of frequency distribution. In the rest of the paper we will assume the value of load mean reversal time $\tau_{p}=30 \mathrm{~s}$ as a base value (unless otherwise is specified). For the load diffusion coefficient we will use $D=6 \cdot 10^{-6} \mathrm{~s}^{-1}$ which provides the value of RMS frequency deviation close to that obtained from the real-life GB system data [24]. We will also assume that the aggregate value of inverse droop provided by generators to be $\alpha_{g}=11$ (this value for GB system is taken from [35]) and assume the load sensitivity to frequency to be 1.5 p.u., which yields a total inverse system droop of $\alpha=\alpha_{g}+\alpha_{L}=12.5$. The base values of the parameters used are summarized in Table [

\section{B. Secondary Frequency Control}

Let us now investigate the role of secondary control on the frequency fluctuations. We assume that secondary control is realized through an integral term, which should be added to the right-hand side of equation (8a). Instead of $(8)$, the system of dynamic equations becomes:

$$
\begin{aligned}
2 H \dot{u} & =-\alpha u-p-K_{i} \theta \\
\dot{\theta} & =u \\
\dot{p} & =-\frac{1}{\tau_{p}} p+b \xi
\end{aligned}
$$

where $K_{i}$ is the secondary control parameter with a typical value around $\sim 0.05 s^{-1}$ [36]. The corresponding probability density function $\mathcal{F}(u, p, \theta)$ is now a function of three variables and has a Gaussian form of (6). Using (5), (17), and (7) one can find the corresponding covariance matrix. After integration over $p$ and $\theta$, similar to (12), the corresponding frequency probability density function $F(u)$ can be obtained. It has 
the same form as 14 with the following value of standard deviation $\sigma$ :

$$
\sigma=\left(\frac{D \tau_{p}^{2}}{\alpha\left(2 H+\alpha \tau_{p}+K_{i} \tau_{p}^{2}\right)}\right)^{1 / 2}
$$

As in the previous case, inertia can be neglected in the denominator of (18) since for realistic scenarios it is always $H \ll \alpha \tau_{p}$. In order to evaluate the role of the secondary control one must compare the second and the third terms in the denominator of 18 .

The combination $\alpha / K_{i}$ can be understood as an effective secondary control timescale $\tau_{s}$. For $\alpha \sim 10-20$ and $K_{i}=$ $0.05 \mathrm{~s}^{-1}$ one has the following estimation:

$$
\tau_{s} \equiv \alpha / K_{i} \sim 200-400 s
$$

If the mean load reversal time $\tau_{p}$ is much less than $\alpha / K_{i}$ then one can neglect the $K_{i}$ term in the denominator of (18); in this case, secondary control does not significantly affect the frequency distribution. The physical meaning of $\tau_{p} \ll \alpha / K_{i}$ is that the load fluctuations change sign well before the secondary control starts to give large enough control signals to generators. The estimation (19) is confirmed by reallife data from [33], where the maximum duration of a number of frequency deviation events was just under 10 minutes.

The opposite limit, when the load mean reversal time is much bigger than the secondary control effective time, i.e. $\tau_{p} \gg \alpha / K_{i}$, one can rewrite the expression (18) in the same form as 15], with $\tau_{s}$ instead of $\tau_{p}$ :

$$
\sigma=\frac{\sqrt{D \tau_{s}}}{\alpha}
$$

In either case inertia does not significantly affect the frequency distribution as long as condition $H \ll \alpha \tau_{p}$ is satisfied.

\section{Turbine Time Constant}

Let us now consider the effects of finite turbine time constants on the frequency distribution. We consider a system with a single turbine section having the time constant $\tau_{T}$. Dynamic equations for this system have the form:

$$
\begin{aligned}
2 H \dot{u} & =p_{m}-p \\
\tau_{T} \dot{p}_{m} & =-p_{m}-\alpha u \\
\dot{p} & =-\frac{1}{\tau_{p}} p+b \xi
\end{aligned}
$$

where $p_{m}$ is the mechanical power supplied by the turbine. Similarly to the previous subsection, one can find the PDF for $u, p$ and $p_{m}-\mathcal{F}\left(u, p, p_{m}\right)$. After integration over $p$ and $p_{m}$ the PDF for frequency $F(u)$ with the following standard deviation $\sigma$ can be found:

$$
\sigma=\left[\frac{D \tau_{p}^{2}\left(\alpha \tau_{T}^{2}+2 H\left(\tau_{p}+\tau_{T}\right)\right)}{2 \alpha H\left(\alpha \tau_{p}^{2}+2 H\left(\tau_{p}+\tau_{T}\right)\right)}\right]^{1 / 2}
$$

Typical values for turbine time-constants are from subsecond to several seconds [1], [36], which is significantly less than the load mean reversal time $\tau_{p}$. Under these conditions one would expect the turbine to have little effect on frequency

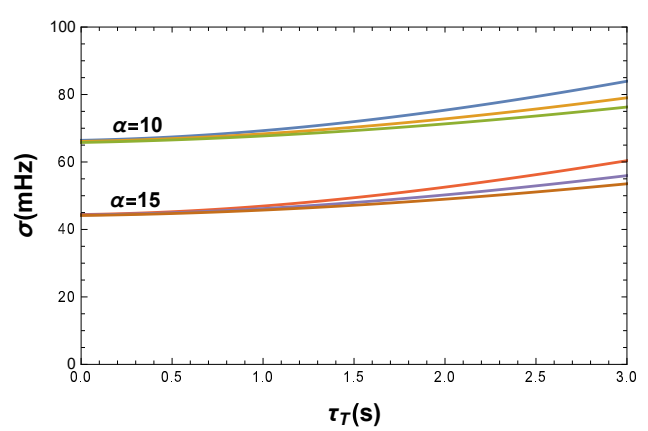

Fig. 3. Root mean square frequency deviation as a function of turbine timeconstant for different values of droop and inertia: the upper and lower groups of curves correspond to inverse droop of $\alpha=10$ and $\alpha=15$ respectively. Three curves in each group correspond to inertia time constants of $H=3 \mathrm{~s}$, $H=4.5 s$ and $H=6 s$ respectively.

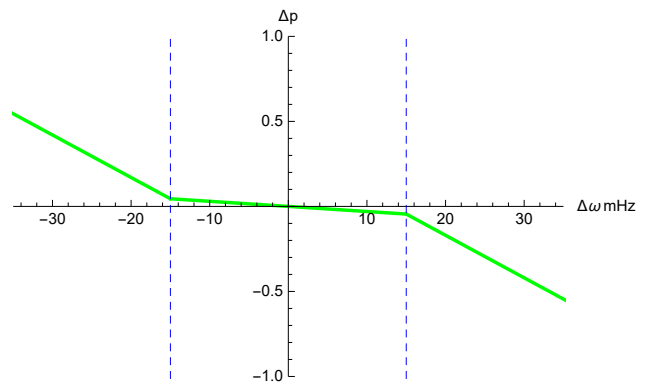

Fig. 4. Power-frequency response of a system in the presence of load sensitivity to frequency (1.5 p.u) and governor deadbands of $\pm 15 \mathrm{mHz}$.

distribution function. Indeed, under the conditions of $\tau_{T} \ll \tau_{p}$ and $\alpha \tau_{T}^{2} \ll H \tau_{p}$ turbine time-constant has a limited influence on the frequency deviation. Fig. 3 gives the frequency standard deviation (in millihertz) as a function of turbine time-constant for different values of droop and inertia. Three curves in each group correspond to three values of inertia $-3 \mathrm{~s}, 4.5 \mathrm{~s}$, and $6 s$ respectively. The upper group (larger frequency deviation) corresponds to aggregate inverse droop of $\alpha=10$, while the lower group corresponds to $\alpha=15$. The load mean reversal time and diffusion coefficients are assumed to be $\tau_{p}=30 \mathrm{~s}$ and $D=6 \cdot 10^{-6} \mathrm{~s}^{-1}$ for all curves.

From Fig. 3 we infer, that the presence of turbine time delay makes the RMS frequency deviation more sensitive to inertia, especially if the inertia itself is small. Specifically, for the value $\tau_{T}=3 \mathrm{~s}$ we find the sensitivity $\nu_{h}=-1.33 \mathrm{mHz}$ for $H=6 \mathrm{~s}$ and $\nu_{h}=-8.98 \mathrm{mHz}$ for $H=2 \mathrm{~s}$. We can claim that the sensitivity of the RMS frequency deviation to system inertia is weak, except in the case of very low inertia. We also note, that this sensitivity depend strongly on the load mean reversal time $\tau_{p}$ (for which we used a rather modest value of $30 \mathrm{~s}$ ), and becomes even smaller with the increase of this parameter.

\section{Governor Deadbands}

In this section we study the effect of deadbands on the frequency PDF. In the presence of governor deadbands the load-frequency response function - denoted here as $\mathcal{K}(u)$ - is no longer linear in $u$, i.e. $\mathcal{K}(u) \neq-\alpha u$. In this subsection 
we consider the Type 2 deadband from [37] where the turbine output is offset by the deadband width. This corresponds to the following value of the response function $\mathcal{K}(u)$ :

$$
\mathcal{K}(u)= \begin{cases}-\alpha\left(u+u_{d b}\right)+\alpha_{L} u_{d b} & u \leq-u_{d b} \\ -\alpha_{L} u & -u_{d b}<u<u_{d b} \\ -\alpha\left(u-u_{d b}\right)-\alpha_{L} u_{d b} & x \geq u_{d b}\end{cases}
$$

where $u_{d b}$ is a per unit deadband - we assume it to be $\pm 15 \mathrm{mHz}=0.0003$ p.u. [38]. Here $\alpha_{g}=11$ p.u. is the generators aggregate inverse droop and $\alpha_{L}=1.5$ p.u. is the load sensitivity coefficient respectively (see Table \). The overall inverse droop is thus $\alpha=\alpha_{L}+\alpha_{g}=12.5$ p.u. The response function (23) is shown in the Fig. 4

The system of dynamic equations corresponding to powerfrequency response 23) can no longer be fully linearized to the form of (5); consequently the corresponding Fokker-Plank equation is also non-linear and formulas (6) and (7) can not be used. However, as was established in the previous subsections, the influence of both secondary frequency control and turbine dynamics on the frequency PDF is small. Therefore, we can consider a 2-dimensional system similar to (8) with power frequency response $\mathcal{K}(u)$ neglecting the secondary control and turbine dynamics altogether:

$$
\begin{aligned}
& 2 H \dot{u}=\mathcal{K}(u)-p \\
& \dot{p}=-\frac{1}{\tau_{p}} p+b \xi
\end{aligned}
$$

The Fokker-Planck equation corresponding to this dynamic system is:

$$
D \frac{\partial^{2} \mathcal{F}}{\partial p^{2}}+\frac{1}{\tau_{p}} \frac{\partial(p \cdot \mathcal{F})}{\partial p}-\frac{1}{2 H} \frac{\partial}{\partial u}[\mathcal{K}(u)-p] \mathcal{F}=0
$$

It is also non-linear due to the presence of the $\mathcal{K}(u)$-term, and can not be solved analytically.

To numerically solve equation (25) we employ the finite element method with static mesh refinement, which enhances the resolution in the region around the deadband - the simulation code has been posted online 11. The contour plot of the PDF for deviations of power and frequency - solution of Fokker-Planck equation (24) is shown in Fig. 5 system parameters are given in Table [I. The distribution is bi-modal and frequency tends to spend most of the time outside the deadbands. Such a bi-modal distribution is fully consistent with experimentally observed data from a number or real-life systems: Great Britain [24] (also given by Fig. 2), Ireleand [16], and Texas [39]. However, we note that the frequency distribution of the continental Europe power is uni-modal [40], [41]. One of the reasons for this could be the requirement for zero intentional deadband [38]. The size of the European grid with it's multiple zones can also contribute to "smoothing" the deadband region since every zone can have it's own frequency response settings.

A practically important question related to frequency control is the influence of deadband width and inertia on the PDF frequency deviations. It is reasonable to assume that as inertia decreases, the amplitude of frequency deviations due to

\footnotetext{
${ }^{1}$ https://github.com/petrvorob/frequencyPDF
}

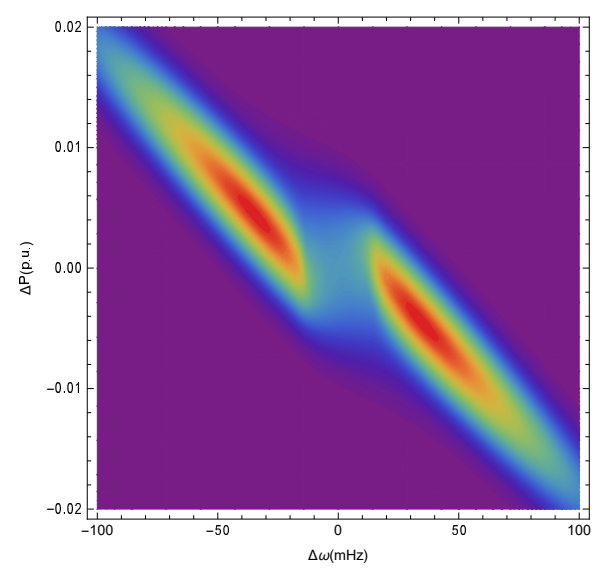

Fig. 5. Contour graph of a simultaneous probability density function of frequency and power deviations - solution of equation 24. Lower frequencies correspond to positive power fluctuations and vice-versa.

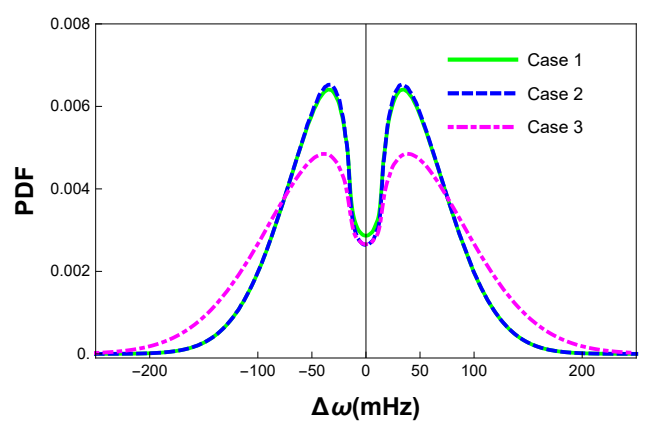

Fig. 6. Effect of power system parameters and primary control settings on the frequency deviations PD. Case 1: $\tau_{p}=30 s, D=6 \cdot 10^{-6} s^{-1}, \alpha=12.5$, Case 2: $\tau_{p}=45 s, D=4 \cdot 10^{-6} s^{-1}, \alpha=12.5$, Case 3: $\tau_{p}=30 s$, $D=6 \cdot 10^{-6} s^{-1}, \alpha=9.2$. For Value of the product $D \tau_{p}$ is the same for all cases.

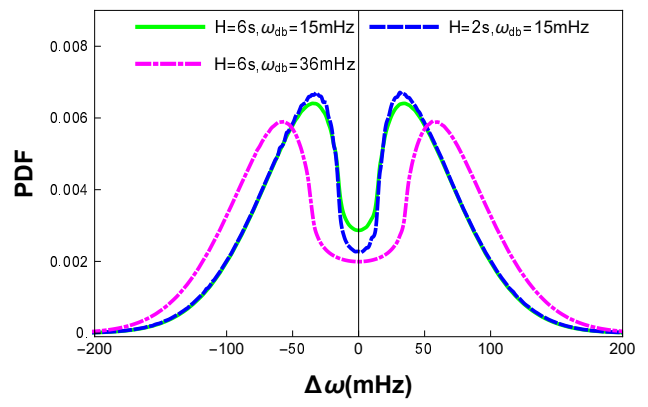

Fig. 7. Effect of deadband width $\left(\omega_{d b}= \pm 15 \mathrm{mHz}\right.$ and $\left.\omega_{d b}= \pm 36 \mathrm{mHz}\right)$ and system inertia ( $H=6 s$ and $H=2 s$ ) on the frequency deviations PDF.

stochastic load fluctuations will increase. Does that mean that the deadbands should be increased in response, to reduce the wear-and-tear of the generator equipment? One of the main arguments related to this is that the choice of deadband significantly affects generator's participation in primary response the so-called governor movement [39]. It was initially assumed that with the narrowing of the deadbands, generators will spend significantly more time in the outer region, thus being under moving governor conditions as opposed to constant power output. In fact, this assumption is not correct as can be seen from Fig 7 where the frequency deviations PDF is given 
for systems with two different deadband sizes - $\pm 15 \mathrm{mHz}$ and $\pm 36 \mathrm{mHz}$. As can be seen, frequency tends to spend most of the time outside of the deadband, irrespective of its width. For the particular example of Fig. 7 numerical evaluation over the corresponding PDFs gives the result that the system is outside of the deadbands about $\approx 89 \%$ of time for the deadband of $\pm 15 \mathrm{mHz}$, and about $\approx 81 \%$ of time for the deadband of $\pm 36 \mathrm{mHz}$. These results are in excellent agreement with the experimental data from ERCOT power system [39], where the simultaneous reduction of deadbands over the whole system from $\pm 36 \mathrm{mHz}$ to $\pm 17 \mathrm{mHz}$ lead to just $5 \%$ increase in generator movement for each unit. Another important conclusion that can be drawn from observing the PDFs in Fig 7 is that system inertia plays very little role, which is a consequence of the fact that $H \ll \alpha \tau_{p}$ and is in full agreement with what was established in Subsection III

An approximate interpretation of the distribution from Fig. 7 is the Gaussian tail on each side of the deadband with the standard deviation still given by the formula (15). To further confirm this interpretation, Fig. 6 shows frequency PDFs for different values of the system parameters. Cases 1 and 2 have the same droop - $\alpha=12.5$ but different values of load mean reversal time and diffusion coefficient $-\tau_{p}=30 \mathrm{~s}, D=6 \cdot 10^{-6}$ $s^{-1}$ and $\tau_{p}=45 \mathrm{~s}, D=4 \cdot 10^{-6} \mathrm{~s}^{-1}$ respectively (so that the product $D \tau_{p}$ is the same for both cases). Case 3 has the lower value of inverse droop $\alpha=9.2$ which can be interpreted as $30 \%$ additional renewable penetration and retirement of the corresponding amount of conventional generation, so that the aggregated primary frequency response becomes weaker. Inertia is assumed to be $H=6 \mathrm{~s}$ for all three cases.

\section{Numerical Evaluation}

\section{A. Linearized model}

Efficient solution of Fokker-Planck equation, described in details in the previous section, is only possible for simplified models with just few degrees of freedom. Neglecting governor deadbands yields linear equations, and closed-form analytic solutions as given by equations (14), (18) and (22) can be obtained. The advantage of these analytic expressions is that they explicitly show the dependence of frequency deviations on every system parameter. Neglecting secondary control and turbine dynamics for a nonlinear system with deadbands allows one to obtain a tractable two-dimensional FokkerPlank equation 25. even for nonlinear frequency response function, for which the solution was presented in the Section III-D In this subsection we present the results of direct time-domain numerical simulations over the linearized onebus model with non-linear frequency response to validate our previous findings. A full nonlinear model will be considered in the next subsection.

As was said, in this subsection we consider the singlebus approximation [27], however, with a full set of dynamic variables, including secondary frequency control, governor

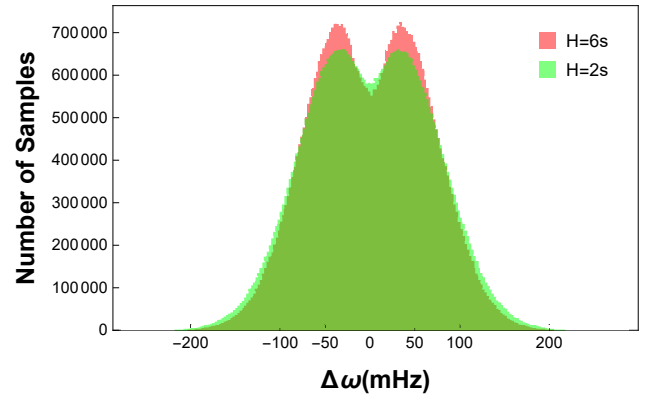

Fig. 8. Histograms of frequency deviations for the system 26 with parameters taken from Table \and two values of inertia time-constant - $H=6 \mathrm{~s}$ red, $H=2 s$ - green.

deadbands, and turbine dynamics:

$$
\begin{aligned}
2 H \dot{u} & =p_{m}-p-K_{i} \theta \\
\dot{\theta} & =u \\
\tau_{T} \dot{p}_{m} & =-p_{m}+\mathcal{K}(u) \\
\dot{p} & =-\frac{1}{\tau_{p}} p+b \xi
\end{aligned}
$$

The base values of power system parameters in 26 are taken from the Table I. unless otherwise specified. Simulations are run for $5 \cdot 10^{5} \mathrm{~s}$ with the time step of $0.01 \mathrm{~s}$. All simulations in this subsection were run on Wolfram Mathematica software using the ItoProcess module 2 .

Fig 8 shows the histograms for frequency deviations for the system (26) for two values of the inertia time-constant $H=6 s$ and $H=2 s$ with the remaining parameters as shown in Table \. We can observe that inertia has a limited effect on the distribution of frequency deviations - lowering inertia threefold only leads to an increase of the RMS frequency deviation from $\sigma=63 \mathrm{mHz}$ for $H=6 \mathrm{~s}$ to $\sigma=67$ $\mathrm{mHz}$ for $H=2$. Thus, the presence of deadbands makes the frequency deviations less sensitive to inertia, compared to the case of Subsection [II-C We also note that the presence of a finite turbine time-constant increases the probability of the frequency being within the deadband region when compared to Fig 7 In fact, the result of Fig 8 is in good agreement with real-life data from Great Britain system shown in Fig 2 It should be noted that the frequency PDF for the Great Britain system is not symmetric, contrary to those obtained through the models in this paper. This is most likely a consequence of the fact that primary reserve is procured separately for low and high frequency events, through services called primary and high frequency response respectively. Mathematically this is equivalent to different slopes of aggregate droop for positive and negative frequency deviations.

Fig 9 illustrates the effect of deadband width, presenting the results of stochastic simulations over the model 26 for two systems with different values of deadband width $- \pm 15 \mathrm{mHz}$ and $\pm 36 \mathrm{mHz}$ respectively (with the rest of parameters taken from Table \. These results are in agreement with the Fig7 confirming the fact that frequency tends to spend most of the time outside the deadbands irrespective of the deadband width.

\footnotetext{
${ }^{2}$ https://github.com/petrvorob/frequencyPDF
} 


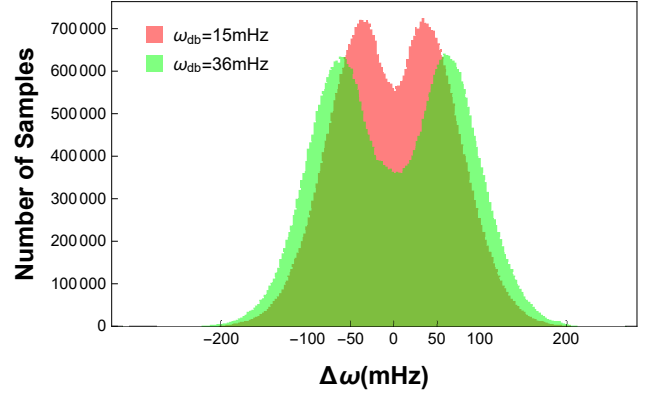

Fig. 9. Frequency PDF for two systems with different deadband width - green color - deadband $\pm 36 \mathrm{mHz}$, red color - deadband $\pm 15 \mathrm{mHZ}$.

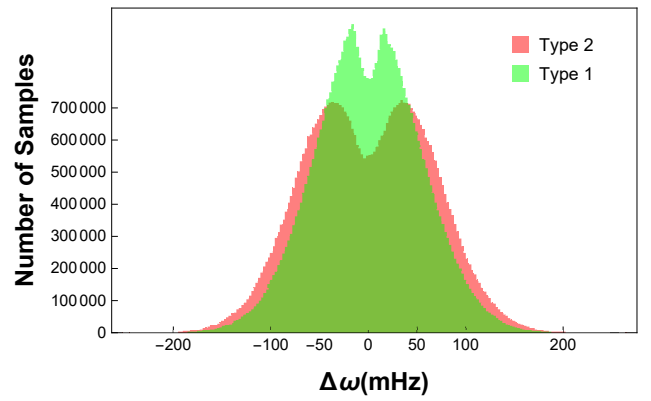

Fig. 10. Frequency PDF for two systems with different types of deadband realization (according to [37], Appendix B). Green color - Type 1 deadband, red color - Type 2 deadband (same as in Fig 4 .

TABLE I

BASIC VALUES OF POWER SYSTEM PARAMETERS

\begin{tabular}{c|c|c} 
Parameter & Description & Value \\
\hline$H$ & Inertia time-constant & $6 \mathrm{~s}$ \\
$\alpha$ & Inverse droop of the system & $12.5 \mathrm{p} \mathrm{u}$ \\
$\omega_{d b}$ & Governor deadband width & $\pm 15 \mathrm{mHz}$ \\
$\tau_{p}$ & Load mean reversal time & $30 \mathrm{~s}$ \\
$D$ & Load diffusion coefficient & $6 \cdot 10^{-6} \mathrm{~s}^{-1}$ \\
$K_{i}$ & Secondary control gain & $0.05 \mathrm{~s}^{-1}$ \\
$\tau_{T}$ & Mean turbine time-constant & $1.5 \mathrm{~s}$ \\
\hline
\end{tabular}

Finally, Fig. 10 shows the frequency distribution for systems with Type 1 and Type 2 deadband realization (according to [37]). Throughout the manuscript we have been using the Type 2 deadband (also shown by Fig. (4) where the frequency response is offset by the deadband. The Type 1 deadband realizes fully liner frequency response with abrupt changes of power output at both ends of the deadband. Since the effective inverse droop of the Type 1 is bigger (provided the droop settings are the same for both types), the frequency deviations are smaller. However, there are concerns about sudden turbine movements at the deadband boundaries for this case [39].

\section{B. Full model}

Let us now verify the findings of the manuscript on a fullscale nonlinear model. As a case study we use a system shown in Fig. 11 which is a modified two-area 14 bus system from [36]. Unlike the simplified model described in the previous subsection, in this case the full non-linear power flows, subtransient generator dynamics, and governor dynamics are in-

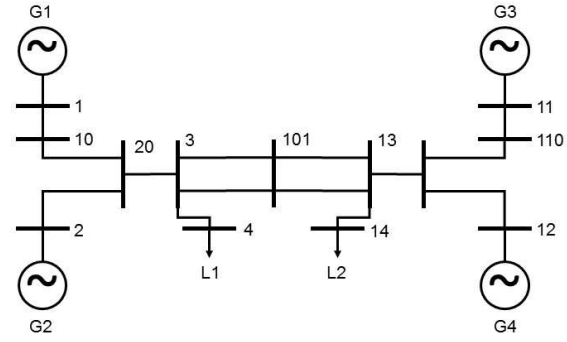

Fig. 11. 14 bus test system from [36] used for non-linear simulations.

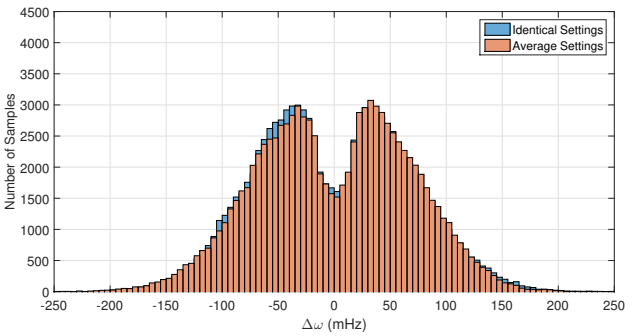

Fig. 12. Frequency PDF for power system of Fig. 11 obtained from simulations over non-linear model with identical generator and load settings (blue) and varied settings (brown).

cluded within the simulation. The simulations were carried out using the Matlab Power Systems Toolbox (PST) [42], which is a standard tool for power system dynamic simulations. The standard PST functions were modified to allow the droop response shown in Fig. 4 including the load response and a generator response with deadband. The secondary control term was implemented using a trapezoidal approximation, without a deadband.

In simulations of this subsection we use the same aggregate parameters as before, given in Table [1 We consider two different cases: first - with uniform parameters of generators and loads, second - with varied parameters keeping the aggregate values same in both cases. The parameters of generators and loads for the second case are given in the Tables [II and III respectively. Fig. 12 shows the frequency PDFs for these two cases. Both histograms show good agreement with the prediction given by the Fokker-Plank equation. The mean absolute percentage error between the two distributions is $0.15 \%$, illustrating the minimal difference between the two cases, suggesting that the single area approximation is acceptable for studying the frequency distribution. Both cases correspond to a $67 \mathrm{mHz}$ RMS frequency deviation which is very close to $63 \mathrm{mHz}$ given by linear model (26) with the same parameters. Fig. 13 illustrates the influence of inertia value on the frequency PDF for the full nonlinear model. We note, that in this case the effect is even smaller, than the simplified linear model gives - lowering inertia from $H=6 \mathrm{~s}$ to $H=2$ $\mathrm{s}$ changed the RMS frequency deviation by less than $1 \mathrm{mHz}$.

Let us now consider the effect of the system loading level on the frequency PDF. We use the actual equilibrium loading level as a base power. Assuming that the relative load fluctuations 
TABLE II

VALUES OF INERTIA, INVERSE DROOP, AND TURBINE TIME-CONSTANT FOR FOUR GENERATORS FOR THE SYSTEM OF FIG.11FOR VARIED SETTINGS

\begin{tabular}{|c|c|c|c|}
\hline Generator & $\mathbf{H}(\mathrm{s})$ & $\alpha_{g}(\mathrm{pu})$ & $\tau_{T}(\mathrm{~s})$ \\
\hline 1 & 8 & 13 & 2 \\
\hline 2 & 10 & 12 & 1.75 \\
\hline 3 & 4 & 10 & 1.25 \\
\hline 4 & 2 & 9 & 1 \\
\hline
\end{tabular}

TABLE III

VALUES OF LOAD MEAN REVERSAL TIME AND DIFFUSION COEFFICIENTS FOR TWO LOADS FOR THE SYSTEM OF FIG.11FOR VARIED SETTINGS

\begin{tabular}{|c|c|c|}
\hline Load & $\tau_{p}(\mathrm{~s})$ & $\mathbf{D}\left(s^{-1}\right)$ \\
\hline 1 & 25 & $7.2 \cdot 10^{-6}$ \\
\hline 2 & 35 & $5.1 \cdot 10^{-6}$ \\
\hline
\end{tabular}

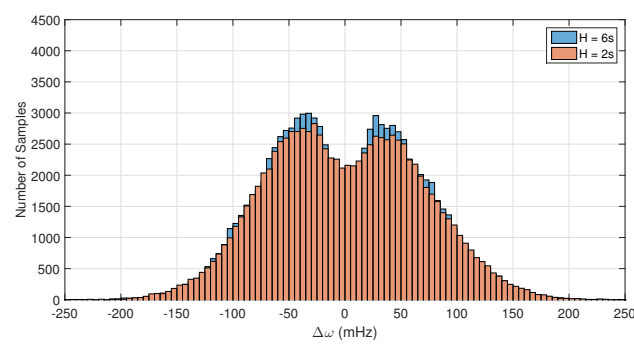

Fig. 13. Frequency PDF for power system of Fig. 11 obtained from simulations over non-linear model for different values of inertia - $H=6$ s (blue) and $H=2 \mathrm{~s}$ (brown).

magnitude is independent of the loading level, all the values for $\tau_{p}$ and $D$ stay unchanged in per unit. However, the inverse droop is proportional to the generator rating rather than the equilibrium loading level (used as a base power), therefore, a reduction in the latter effectively corresponds to an increase in the per unit droop response, unless generating units are being brought off-line in response to load decrease, thus reducing the aggregate response. Similarly, since inertia time constant $H$ is related to base power, reducing the loading level also results in an increase in total system inertia. The impact of changing the loading level on the frequency PDF is shown by Fig. 14, with histograms of the loading levels of $100 \%$ and $70 \%$. The lower loading results in a narrower histogram, and leads to smaller RMS frequency deviation ( $51 \mathrm{mHz}$ vs $67 \mathrm{mHz}$ ). However, as was mentioned before, this is only true under the assumption that all the generating units stay online with the same droop settings despite the reduction in overall loading level.

\section{Conclusions}

We have studied the power system frequency distribution in the presence of ambient load fluctuations. We considered the effects of different system parameters on the standard deviation of frequency; the following results can be summarized:

1) Role of inertia and turbine time constant. Under the assumption (confirmed by real-life data) that the mean load reversal time $\tau_{p}$ is at least few decades of seconds,

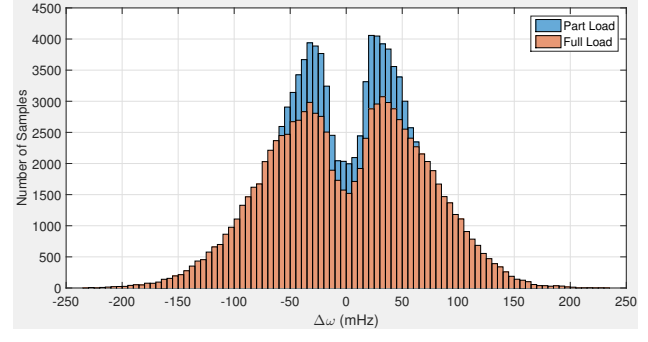

Fig. 14. Frequency PDF for power system of Fig. 11 obtained from simulations over non-linear model with different loading levels: blue - $100 \%$ load, brown - $70 \%$ load.

both inertia and turbine time constants have minimal impact on the frequency standard deviation. This is in contrast to the case of frequency response to major contingencies, where exactly these two parameters are of crucial importance because they determine the size of the transient frequency dip. On the one hand, this suggests that reduced system inertia will not cause the increase of the RMS frequency deviation on it's own. However, inertia can be related to some other important parameters, such as aggregate droop. In this case there could be the correlation between the decrease of inertia and the increase of the frequency fluctuations (see discussion on renewables below). Another important conclusion is that procuring synthetic inertia will not help improving the RMS frequency deviation and some other measures have to be taken.

2) Role of governor deadbands. The width of governor deadbands has a major effect on the frequency distribution. Frequency tends to spend most of the time outside the deadband region, regardless of its width. This suggests that there does not exist any "optimal" deadband size, that could substantially reduce wear-andtear of generator equipment due to its movement. The presence of deadbands results in a bi-modal frequency distribution, which is in excellent agreement with reallife frequency data from a number of systems [16], [24], [39].

3) Role of aggregated droop. The aggregate system droop is the major parameter determining the standard frequency deviation (eq.15). Increasing renewable penetration, and the corresponding displacement of conventional generators, can lead to a reduction in the aggregate system inverse droop $\alpha$, which will, correspondingly, cause larger frequency deviations. One of the ways to deal with the problem is to increase the inverse droop coefficients of the remaining generators; however, this could be limited by stability considerations. Use of energy storage for frequency control could be a solution, since storage can potentially provide almost deadbandless and delay-less frequency response [34].

4) Role of renewables penetration. Renewable generation can bring a number of simultaneous effects to power system. Reduction of the system inertia is usually cited as the major one, however, renewable generation could also increase the effective load diffusion coefficient $D$, 
as well as decreasing the system-wide inverse droop due to some conventional generation being brought offline and assuming renewable generators do not participate in frequency response. Overall, the increased penetration of renewable sources can lead to substantial increase of the RMS frequency deviation. According to our results this problem can not be resolved by procuring synthetic inertia. The latter can greatly improve the system transient response following major contingencies, but will have minimal effect on the ambient frequency fluctuations, so that some additional measures have to be taken. Demanding renewables participation in frequency response on a continuous basis could be one of such measures.

\section{REFERENCES}

[1] J. Machowski, J. Bialek, and J. Bumby, Power system dynamics: stability and control. John Wiley \& Sons, 2011.

[2] J. O'Sullivan, A. Rogers, D. Flynn, P. Smith, A. Mullane, and M. O'Malley, "Studying the maximum instantaneous non-synchronous generation in an island systemfrequency stability challenges in ireland," IEEE Trans. Power Syst., vol. 29, no. 6, pp. 2943-2951, 2014.

[3] G. Lalor, J. Ritchie, S. Rourke, D. Flynn, and M. J. O'Malley, "Dynamic frequency control with increasing wind generation," in Power Engineering Society General Meeting, 2004. IEEE. IEEE, 2004, pp. $1715-1720$.

[4] S. Püschel-L, P. Mancarella et al., "Mapping the frequency response adequacy of the australian national electricity market," in Universities Power Engineering Conference (AUPEC), 2017 Australasian. IEEE, 2017, pp. 1-6.

[5] F. Teng, V. Trovato, and G. Strbac, "Stochastic scheduling with inertiadependent fast frequency response requirements," IEEE Trans. Power Syst., vol. 31, no. 2, pp. 1557-1566, 2016.

[6] A. Ulbig, T. S. Borsche, and G. Andersson, "Impact of low rotational inertia on power system stability and operation," IFAC Proceedings Volumes, vol. 47, no. 3, pp. 7290-7297, 2014.

[7] S. EirGrid, "All island tso facilitation of renewables studies," Eirgrid, SONI, Dublin, 2010.

[8] P. Tielens and D. Van Hertem, "The relevance of inertia in power systems," Renewable and Sustainable Energy Reviews, vol. 55, pp. 999 1009, 2016.

[9] R. G. De Almeida and J. P. Lopes, "Participation of doubly fed induction wind generators in system frequency regulation," IEEE Trans. Power Syst., vol. 22, no. 3, pp. 944-950, 2007.

[10] D. Gautam, L. Goel, R. Ayyanar, V. Vittal, and T. Harbour, "Control strategy to mitigate the impact of reduced inertia due to doubly fed induction generators on large power systems," IEEE Trans. Power Syst., vol. 26, no. 1, pp. 214-224, 2011.

[11] F. Teng and G. Strbac, "Evaluation of synthetic inertia provision from wind plants," in Power \& Energy Society General Meeting, 2015 IEEE. IEEE, 2015, pp. 1-5.

[12] N. Jaleeli and L. S. VanSlyck, "Nerc's new control performance standards," IEEE Trans. Power Syst., vol. 14, no. 3, pp. 1092-1099, 1999.

[13] Nerc: Balancing and frequency control. [Online]. Available: https://www.nerc.com/docs/oc/rs/NERC\%20Balancing\%20and \%20Frequency\%20Control\%20040520111.pdf

[14] F. Milano and R. Zárate-Miñano, "A systematic method to model power systems as stochastic differential algebraic equations," IEEE Trans. Power Syst., vol. 28, no. 4, pp. 4537-4544, 2013.

[15] C. Roberts, E. M. Stewart, and F. Milano, "Validation of the ornsteinuhlenbeck process for load modeling based on $\mu$ pmu measurements," in Power Systems Computation Conference (PSCC), 2016. IEEE, 2016, pp. 1-7.

[16] F. M. Mele, A. Ortega, R. Zárate-Minano, and F. Milano, "Impact of variability, uncertainty and frequency regulation on power system frequency distribution," in Power Systems Computation Conference (PSCC), 2016. IEEE, 2016, pp. 1-8.

[17] P. Huynh, H. Zhu, Q. Chen, and A. Elbanna, "Data-driven estimation of frequency response from ambient synchrophasor measurements," IEEE Trans. Power Syst., 2018.
[18] H. Chavez, R. Baldick, and S. Sharma, "Regulation adequacy analysis under high wind penetration scenarios in ercot nodal," IEEE Trans. Sustainable Energy, vol. 3, no. 4, pp. 743-750, 2012.

[19] H. Chávez, R. Baldick, and J. Matevosyan, "Cps1 complianceconstrained agc gain determination for a single-balancing authority," IEEE Trans. Power Syst., vol. 29, no. 3, pp. 1481-1488, 2014.

[20] H. Chvez, R. Baldick, and J. Matevosyan, "The joint adequacy of agc and primary frequency response in single balancing authority systems," IEEE Trans. on Sustainable Energy, vol. 6, no. 3, pp. 959-966, 2015.

[21] K. Wang and M. L. Crow, "The fokker-planck equation for power system stability probability density function evolution," IEEE Trans. Power Syst., vol. 28, no. 3, pp. 2994-3001, 2013.

[22] E. Mallada, "idroop: A dynamic droop controller to decouple power grid's steady-state and dynamic performance," in Decision and Control (CDC), 2016 IEEE 55th Conference on. IEEE, 2016, pp. 4957-4964.

[23] T. Sasaki and K. Enomoto, "Statistical and dynamic analysis of generation control performance standards," IEEE Trans. Power Syst., vol. 17, no. 2, pp. 476-481, 2002.

[24] "National grid. frequency data for 2016." [Online]. Available: https:// www.nationalgrid.com/uk/electricity/balancing-services/frequencyresponse-services/firm-frequency-response?market-information

[25] H. Risken, the Fokker-Plnack Equation: Methods of Solution and Applications. New York: Springer, 1989.

[26] F. Paganini and E. Mallada, "Global performance metrics for synchronization of heterogeneously rated power systems: The role of machine models and inertia," arXiv preprint arXiv:1710.07195, 2017.

[27] J. O'Sullivan, M. Power, M. Flynn, and M. O'Malley, "Modelling of frequency control in an island system," in Power Engineering Society 1999 Winter Meeting, IEEE, vol. 1. IEEE, 1999, pp. 574-579.

[28] G. Lalor, J. Ritchie, D. Flynn, and M. J. O'Malley, "The impact of combined-cycle gas turbine short-term dynamics on frequency control," IEEE Trans. Power Syst., vol. 20, no. 3, pp. 1456-1464, 2005.

[29] B. Øksendal, "Stochastic differential equations," in Stochastic differential equations. Springer, 2003, pp. 65-84.

[30] G. Ghanavati, P. D. Hines, and T. I. Lakoba, "Identifying useful statistical indicators of proximity to instability in stochastic power systems," IEEE Trans. Power Syst., vol. 31, no. 2, pp. 1360-1368, 2016.

[31] P. M. Ashton, C. S. Saunders, G. A. Taylor, A. M. Carter, and M. E. Bradley, "Inertia estimation of the gb power system using synchrophasor measurements," IEEE Trans. Power Syst., vol. 30, no. 2, pp. 701-709, 2015.

[32] F. Teng and G. Strbac, "Assessment of the role and value of frequency response support from wind plants," IEEE Trans. Sustainable Energy, vol. 7, no. 2, pp. 586-595, 2016.

[33] Nerc load-generation and reserves reliability control standards project. [Online]. Available: https://www.energy.gov/sites/prod/files/2016/06/ f32/20.\%20Martinez\%20Load\%20Generating\%20Reserves.pdf

[34] D. Greenwood, K. Y. Lim, C. Patsios, P. Lyons, Y. S. Lim, and P. Taylor, "Frequency response services designed for energy storage," Applied Energy, vol. 203, pp. 115-127, 2017.

[35] J. B. Ekanayake, N. Jenkins, and G. Strbac, "Frequency response from wind turbines," Wind Engineering, vol. 32, no. 6, pp. 573-586, 2008.

[36] P. Kundur, N. J. Balu, and M. G. Lauby, Power system stability and control. McGraw-hill New York, 1994, vol. 7.

[37] P. Pourbeik et al., "Dynamic models for turbine-governors in power system studies," IEEE Task Force on Turbine-Governor Modeling, no. 2013, 2013.

[38] Y. G. Rebours, D. S. Kirschen, M. Trotignon, and S. Rossignol, "A survey of frequency and voltage control ancillary servicespart i: Technical features," IEEE Trans. Power Syst., vol. 22, no. 1, pp. 350357, 2007.

[39] I. Abdur-Rahman, "Frequency regulation: is your plant compliant? introducing wind and solar into the grid highlights the importance of optimizing power plant frequency regulation capabilities," Power Engineering, vol. 114, no. 10, pp. 66-73, 2010.

[40] B. Polajžer, D. Dolinar, and J. Ritonja, "Analysis of ace target level for evaluation of load frequency control performance," in Energy Conference (ENERGYCON), 2016 IEEE International. IEEE, 2016, pp. 1-4.

[41] "Entso-e supporting document for the network code on load-frequency control and reserves, june 2013." [Online]. Available: https://www. acer.europa.eu/Official_documents/Acts_of the_Agency/Annexes/ ENTSO-E\%E2\%80\%99s\%20supporting\%20document $\% 20$ to\%20the $\% 20$ submitted $\% 20$ Network\%20Code $\% 20$ on $\% 20$ Load-Frequency $\% 20$ Control\%20and\%20Reserves.pdf

[42] J. H. Chow and K. W. Cheung, "A toolbox for power system dynamics and control engineering education and research," IEEE transactions on Power Systems, vol. 7, no. 4, pp. 1559-1564, 1992. 


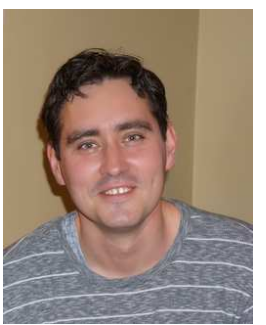

Petr Vorobev (M`15) received his Ph.D. degree in theoretical physics from Landau Institute for Theoretical Physics, Moscow, in 2010. Currently, he is an Assistant Professor at Skolkovo Institute of Science and Technology (Skoltech), Moscow, Russia. Before joining Skoltech, he was a Postdoctoral Associate at the Mechanical Engineering Department of Massachusetts Institute of Technology (MIT), Cambridge. His research interests include a broad range of topics related to power system dynamics and control. This covers low frequency oscillations in power systems, dynamics of power system components, multi-timescale approaches to power system modelling, development of plug-and-play control architectures for microgrids.

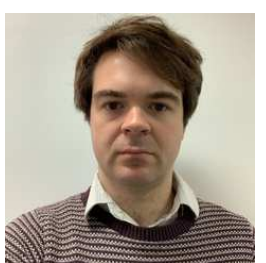

David M. Greenwood (M14) received the M.Eng. degree in new and renewable energy from Durham University, Durham, U.K., in 2010 and the Ph.D. degree, for work on real-rime thermal ratings, from Newcastle University, Newcastle upon Tyne, U.K., in 2014. He is currently a Senior Research Associate at Newcastle University. His research focusses on flexibility services the role of energy storage within the power system.

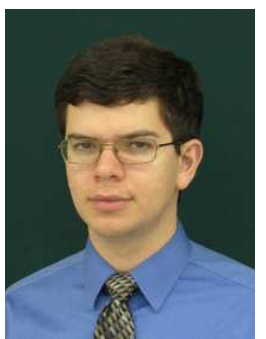

John H. Bell received his S.B. degree in mechanical engineering from the Massachusetts Institute of Technology (MIT), Cambridge, in 2018. Currently, he is a masters student in the Mechanical Engineering Department of MIT. His current research interests primarily consist of the dynamics, control, and design of robotic and electromechanical systems. This covers topics such as motor control, gait control for legged robots, and coordination of compound robot-human systems.

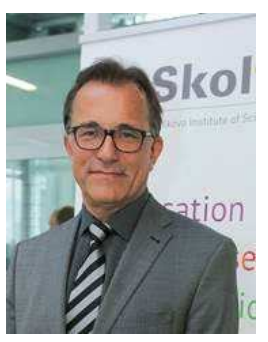

Janusz W. Bialek (F11) received M.Eng. (1977) and Ph.D. (1981) degrees from Warsaw University of Technology, Warsaw, Poland. He is Founding Director of the Center for Energy Systems, Skolkovo Institute of Science and Technology (Skoltech), Russia, having previously held Chair positions at The University of Edinburgh (20032009), and Durham University (20092014). His interests are in smart grids, energy system integration, power system dynamics, power system control, power system economics, and preventing electricity blackouts.

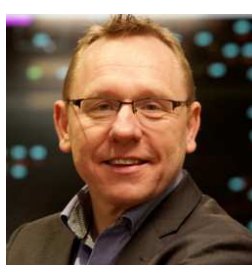

Philip C. Taylor (SM12) received the Engineering Doctorate in the field of intelligent demand side management techniques from the University of Manchester Institute of Science and Technology (UMIST), Manchester, U.K., in 2001. He joined Newcastle University, Newcastle upon-Tyne, U.K., in April 2013 where he is the Head of the School of Engineering and holds the Siemens Chair of Energy Systems. He is a visiting Professor at Nanyang Technological University in Singapore and he previously held the DONG Energy Chair in Renewable Energy and was a Director of the Durham Energy Institute.

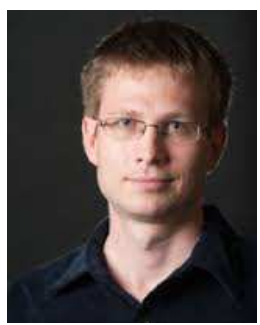

Konstantin Turitsyn (M'09) received the M.Sc. degree in physics from Moscow Institute of Physics and Technology and the Ph.D. degree in physics from Landau Institute for Theoretical Physics, Moscow, in 2007. He was an Associate Professor at the Mechanical Engineering Department of Massachusetts Institute of Technology (MIT), Cambridge. Before joining MIT, he held the position of Oppenheimer fellow at Los Alamos National Laboratory, and KadanoffRice Postdoctoral Scholar at University of Chicago. His research interests encompass a broad range of problems involving nonlinear and stochastic dynamics of complex systems. Specific interests in energy related fields include stability and security assessment, integration of distributed and renewable generation. 